\title{
Part II: U.S.-Sub-Saharan Africa Educational Partnerships for Medical Device Design
}

\section{INTRODUCTION}

Three factors have driven the establishment of engineering design collaborations between U.S. and Sub-Saharan Africa universities that have also contributed to the development of biomedical engineering design capacity for resource-limited settings. First is the need for low cost and robust health technology solutions in Sub-Saharan Africa, which is unparalleled worldwide. Second, is the emergence of bachelor's level degree granting biomedical engineering (BME) programs in Sub-Saharan Africa, which was the focus of Part I of this two part series. Third, is the recognition that co-creative design processes involving local stakeholders generate the most effective global health technology solutions. These three factors have created a collegial base for BME students and faculty in U.S. and Sub-Saharan institutions to engage cooperatively in the design of appropriate technical solutions for global health.

Medical device design is an attractive avenue for establishing meaningful partnerships with Sub-Saharan BME because: the need is significant, the educational value of designing subject to constrained resources, and the provision of a good middle ground that emphasizes resourcefulness and creativity rather than technical sophistication. To highlight this trend the Education Track for the 2016 BMES annual meeting organized a session of invited speakers entitled Global Health Engineering 2.0: Building Educational Capacity in Africa that featured illustrative BME design programs from the U.S., Nigeria and South Africa that have embraced the importance of developing co-creative design partnerships (Figs. 1 and 2). In this installment the most significant facets of each educational partnership are described.

The focus of Part II is summarizing the key characteristics of the illustrative global health design programs at Rice University, University of Michigan, Case Western Reserve University, Northwestern University/Cape Town University, and Duke University, and provides recommendations to U.S. institutions for entering sustainable BME design-focused partnerships.

\section{ILLUSTRATIVE BME DESIGN PARTNERSHIPS WITH SUB-SAHARAN AFRICA}

The Rice University $360^{\circ}$ Institute for Global Health started in 2007 by Professors Rebecca Richards-Kortum and Maria Oden, is arguably the most comprehensive educational program in the immersive co-designing of global healthcare technology in high-income countries. ${ }^{22}$ Richards-Kortum and session keynote speaker Maria Oden gave a joint TEDx talk on their global health design efforts in Malawi. ${ }^{24}$ At Rice, undergraduates of any major may apply to the Global Health Technologies program to work on design problems identified by healthcare partners such as the University of Malawi Polytechnic and College of Medicine and Barretos Cancer Hospital in Brazil. ${ }^{8}$ Students in this program may also intern in their international health setting to implement their technologies. ${ }^{23}$ Students and faculty at both universities benefit from exchange programs that bring members of both universities to each other's campus. International design teams have been responsible for several new health technologies, the most notable being the low-cost bubble continuous positive airway pressure (CPAP) for neonates, a low-cost syringe pump that uses mechanical energy to last between intermittent power outages, a diagnostic device for postpartum hemorrhage, a hand-powered centrifuge made of a salad spinner, an electronic medical records system, and an alternative consumable to test for anemia that costs less than $1 e^{4,5,18,19,25,29}$ The Rice BME faculty has also collaborated with the Malawi faculty to build the undergraduate BME program at the University of Malawi, which is a program in the Department of Engineering. . $^{17,28}$

Kathleen Sienko, Associate Professor of Mechanical Engineering and BME at the University of Michigan, is Director of the Global Health Design Initiative (GHDI) and Co-Director of the Center for Socially Engaged Design. ${ }^{7,28}$ The GHDI provides engineering and non-engineering students with credit-bearing and internship opportunities centered on global health needs assessments and co-creative and context-appropriate design experiences at international and domestic field sites. Prior to entering the clinical immersion sites, students learn the tools of design ethnography that they will employ to identify and define unmet needs and characterize use contexts. ${ }^{21}$ Since 2011, cohorts of engineering students from $\mathrm{U}$ of $\mathrm{M}$ and Ghana have performed needs assessments during their clinical immersion experience primarily at Korle Bu Teaching Hospital. Upon returning to their universities, students generate concept solutions, and fabricate and evaluate 


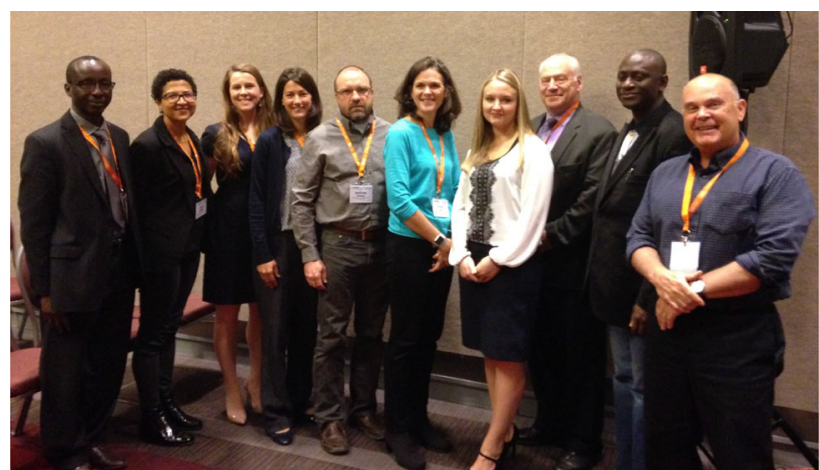

FIGURE 1. Participants in the Global Health Engineering 2.0 session. Symposium speakers are highlighted in bold type. From left: Akinniyi Osuntoki, Tania Douglas, Maria Young, Kathleen Sienko, Andrew Rollins, Maria Oden, Brittany Ploss, Matthew Glucksberg, Akinwale Coker, and session organizer William Reichert.

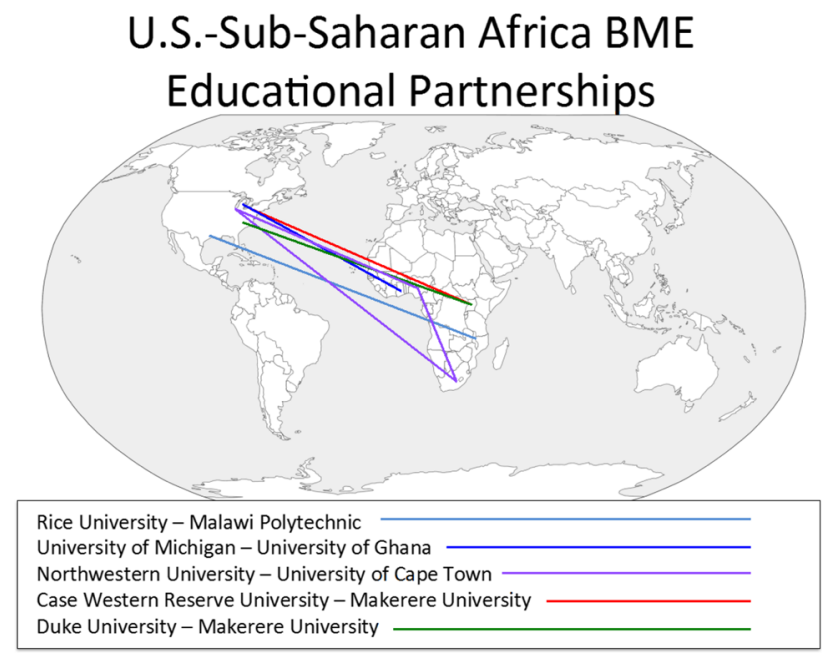

FIGURE 2. Map of educational partnerships. Modified freeware from http://www.waterproofpaper.com/printable-maps/ world/map-of-the-world.pdf.

prototypes. ${ }^{27}$ These projects have included a portable pelvic examination table, and a subdermal contraceptive insertion device that have generated conference abstracts, journal articles, and device commercialization (e.g., Hemafuse). Sienko describes the early work of GHDI in Ghana, Kenya and Uganda in her TEDx talk. ${ }^{26}$

Matthew Glucksberg is the Director of the Center for Innovation in Global Health Technologies at Northwestern University. ${ }^{20}$ Glucksberg has collaborated with BME Professor Tania Douglas of the University of Cape Town (UCT) to coordinate a BME consortium that includes two Nigerian universities: the University of Ibadan (UI) and the University of Lagos (UL). The Nigerian programs, led by Professors
Akinwale Coker and Akinniyi Osuntoki, respectively, are developing curricula that emphasize user-centered design and development supported by expertise from the faculties of medicine and business. This consortium has been convening since 2013 with the aid of an NIH Fogarty International Center training grant (D43TW009374; PI: Robert Murphy), and is engaged in faculty exchanges and the cultivation of academic biomedical engineering in Nigeria. The group is developing a BME textbook for the African context and is launching journal named Global Health Innovation to disseminate knowledge on social and technological innovation for improved health, particularly in developing contexts. Inter-institutional projects in this group have included work on infant surgical warmers, burn injuries, and a blood management system. Visits of the Nigerian faculty to UCT (four to six faculty per annum, staying for six to ten weeks) include participation in courses on design and healthcare technology management. This group has also piloted a short clinical immersion program between students at the Nigerian universities and Northwestern for design prototyping. Northwestern University hosts two or three visiting faculty each year to help develop a design-based BME curriculum. Faculty members of each participating university from the schools of engineering, business and medicine have been tapped to cultivate "locally-grown" healthcare solutions in Nigeria. ${ }^{16}$

Case Western Reserve University (CWRU) has enjoyed a formal multidisciplinary collaboration with Makerere University in Kampala, Uganda since 1988 to fight the AIDS and TB epidemics. In 2011, Makerere University School of Biomedical Sciences admitted its first class of BME undergraduates. ${ }^{3}$ CWRU Professors Andrew Rollins (BME) and Janet McGrath (Anthropology) used this confluence of programs to develop a cross-disciplinary course called "Interdisciplinary Solutions to Global Health Issues" that immerses both BME and Anthropology students interested in global health in the process of design, implementation and anthropology of global health technologies, which includes a spring break trip to Uganda. ${ }^{2}$ The class, which was offered for the first time in the spring of 2015, brings CWRU and Makerere students together to work on ongoing design projects focused in three areas of unmet need: the vaccine cold chain, medical waste management, and pediatric pulse oximetry. ${ }^{1}$ Students at both universities work collaboratively on these design projects, including fieldwork in Luweero, Uganda about 35 miles north of Kampala. This combination of engineering design, anthropology, sustained collaboration with local design teams, and continuance of projects from year to year is a novel and compelling vehicle for teaching the process and nuances of designing tech- 
nology for low-resource contexts. The integration of teams between CWRU and Makerere University also models sustainable partnerships that contribute to capacity building.

The newest program at the session is directed by Duke University Professor of BME William Reichert, who spent the 2014-2015 academic year as a Fulbright Scholar at Makerere University in Kampala (MUK), Uganda. Reichert taught several BME classes to MUK students over two semesters, and conducted a thorough curricular review that resulted in a significant increase in engineering content during the first and second years of the program. Reichert also observed that the number of MUK BME instructors were insufficient to sustain adequate curricular coverage. Upon returning to Duke, Reichert and Duke BME chair Ashutosh Chilkoti launched the Duke-MUK BME Partnership with generous support from the university. ${ }^{10}$ Duke-MUK is taking a four pronged approach: teaching BME classes taken simultaneously by Duke and Makerere students, offering a class where Duke and Makerere BME students work collaboratively on global health design projects originating from Makerere (which includes a Duke spring break trip to Uganda), an Engineering World Health Summer Institute in Uganda, and offering full scholarships to Makerere BME graduates to study for a Master's degree in BME at Duke. Due to Internet resource challenges at MUK, Duke-MUK also set up a BME video conferencing facility at Makerere to facilitate communication. The Duke-MUK scholarship comes with a payback agreement where the scholars must return to Makerere to teach BME classes for at least three semesters. The first two scholars started their studies in August 2016 and plan to finish in December 2017. ${ }^{9}$

Finally, much of the need in LMICs lies in relatively straightforward maintenance and repair of routine equipment that does not require Bachelor-level of training in BME. Dr. Malkin (Duke University), cofounder of Engineering World Health (EWH) ${ }^{12}$ developed a curriculum to train and deploy undergraduates from mostly Europe and North America to LMIC hospitals and clinics during their summer break to maintain and repair medical equipment. Brittany Ploss presented her work with Malkin on the benefits of extending EWH training to local staff that become certified as Biomedical Engineering Technicians (BMETs). EWH provides open-source curricular materials for other institutions that may want to begin a BMET curriculum. ${ }^{13}$ The training programs usually award students a diploma (analogous to an associate's degree) after one to two years of coursework. Locally owned BMET programs with an EWH-based curriculum now exist in Rwanda and Honduras, while schools in Ghana, Cambodia, Nigeria, and Ethiopia are in various stages of ownership transition. ${ }^{15}$ A one-year post-transition monitoring visit by Duke to Honduras demonstrated significant educational impact for the technicians, all of whom were still in the public sector. Hospitals with an EWH trained BMET (compared to a non-EWH trained BMET) had $35 \%$ less out-of-service equipment. This visit also revealed sustained educational efforts by the program, with ongoing program activities more than a year after the EWH funding had ended. ${ }^{11}$

\section{RECOMMENDATIONS}

The most effective BME education for medical device design for global health possesses mutually beneficial partnerships between institutions in high- and low-income countries. Any U.S. university with a global health program has multiple such relationships. Also consider contacting the university's international affairs office. These inquiries can result in a network of established interactions that can be used to start building a relationship.

The next step is to consider how deeply immersed into this relationship one should become. Clearly, the more immersed one gets the greater is the potential for mutually beneficial impact. Listed below in approximate order of increasing effort and cost, are suggestions of actions to consider when building a cooperative relationship with a BME program that would benefit from capacity building efforts.

1. Make available existing course material such as lecture notes, homework solutions, videos, software, and lab exercises. The priority should be courses in device design, such as electrical circuits, computer software, modeling and simulation, medical instrumentation, clinical engineering, biomechanics and rehabilitation.

2. Use distance-learning technology to offer classes at both institutions by delivering streamed or recorded lectures. Some resource investment here may be necessary (e.g. modems, data plans, and visualization technology).

3. Offer existing or new online courses that cover priority BME topics. This requires the same technology considerations as recommendation 2 above.

4. Assist in starting an EWH chapter at the partner institution that specifically links with the EWH chapter in one's own university. EWH chapters are pervasive within BMES as evidenced by the student design awards given each year at the BMES annual meeting. ${ }^{14}$

5. Bring BME students from the partner institution to one's own university using existing research experience for undergraduates (REU) programs. 
6. Build in visits of one's students to the partner institution around a course or internship where students at both institutions participate.

7. Work with the partner institution teaching staff to develop or revise their BME curriculum if needed. It is important to be aware of differences in curricular expectations so that the instituted revisions are an appropriate upgrade.

8. Bring students from the partner institution to study at one's own university longer term. This poses significant challenges from the cost of attending to the preparation mismatch.

9. Partner with a university to establish a design program that takes co-originated ideas from concept to prototypes, and even to manufacturing and distribution when possible, including shared intellectual property considerations.

10. Use these educational partnerships to build research collaborations that address problems significant to the partner institution. From a university perspective, this may be the highest order form of interaction, as it requires taking on commitments for experimental tasks, funds for personnel costs, research supplies and equipment. ${ }^{6}$

\section{REFERENCES}

\footnotetext{
${ }^{1}$ Anthropology, engineering students collaborate on global health issues in Uganda. Case Western Reserve University: the daily. http://thedaily.case.edu/anthropology-engineeringstudents-collaborate-on-global-health-issues-in-uganda/, 2016. ${ }^{2}$ Anthropology, engineering students work together to solve global health problems. Case Western Reserve University. http://engineering.case.edu/engineering-anthropology-globalhealth, 2017.

${ }^{3}$ Bachelor of Science in Biomedical Engineering. Makerere University. https://courses.mak.ac.ug/programmes/bachelorscience-biomedical-engineering- 0 .

${ }^{4}$ Bond, M., C. Elguea, J. S. Yan, M. Pawlowski, J. Williams, A. Wahed, M. Oden, T. S. Tkaczyk, and R. Richards-Kortum. Chromatography paper as a low-cost medium for accurate spectrophotometric assessment of blood hemoglobin concentration. Lab. Chip. 13:2381-2388, 2013.

${ }^{5}$ Brown, J., L. Theis, L. Kerr, N. Zakhidova, K. O'Connor, M. Uthman, Z. M. Oden, and R. Richards-Kortum. A hand-powered, portable, low-cost centrifuge for diagnosing anemia in low-resource settings. Am. J. Trop. Med. Hyg. 85:327-332, 2011.

${ }^{6}$ Carbonnier, G. K., T. North-South Research Partnership: Academia Meets Development? European Association of Development Research and Training Institutes, 2014.

${ }^{7}$ Center for Socially Engaged Design: Program in Global Health Design. University of Michigan. https://csed.engin. umich.edu/program-in-global-health-design/.
}

${ }^{8}$ Department of Bioengineering: Beyond Traditional Borders. Rice University. http://bioengineering.rice.edu/Content. aspx?id $=98,2010$.

${ }^{9}$ Duke Establishes Biomedical Engineering Partnership with Makerere University. Duke University: http://pratt. duke.edu/news/duke-establishes-biomedical-engineeringpartnership-makerere-university, 2016.

${ }^{10}$ Duke-Makerere BME Partnership. https://sites.duke.edu/ dukemuk/.

${ }^{11}$ Emmerling, D., P.W. Sholar, and R.A. Malkin. A Sustainability Evaluation of a Biomedical Technician Training Program in Honduras. Unpublished: 2017.

${ }^{12}$ EWH. Engineering World Health. www.ewh.org, 2017.

${ }^{13}$ EWH. Biomedical Equipment Technician (BMET) Library. http://library.ewh.org/.

${ }^{14}$ EWH. Engineering World Health Design Competition. http://www.ewh.org/students/design-competition/designcompetition.

${ }^{15}$ EWH. Personal Communication. edited by B. Ploss, 2017.

${ }^{16}$ Gatchell, D. W., R. Linsenmeier, R. L. Murphy, A. O. Coker, and A. A. Osuntoki. Developing innovative interdisciplinary biomedical engineering programs in Nigeria: Lessons learned. In: ASEE 2016 International Forum, 2016.

${ }^{17}$ Green, A. Global Health Technology Fellowship: Strengthening Biomedical Engineering Education in Malawi. Florida International University. https://bme.fiu.edu/ 2014/10/rice-university-global-health-technology-fellowshipstrengthening-biomedical-engineering-education-in-malawi/, 2014

${ }^{18}$ Juarez, A., K. Maynard, E. Skerrett, E. Molyneux, R. Richards-Kortum, Q. Dube, and Z. M. Oden. AutoSyP: a low-cost, low-power syringe pump for use in low-resource settings. Am. J. Trop. Med. Hyg. 95:964-969, 2016.

${ }^{19}$ Kawaza, K., H. E. Machen, J. Brown, Z. Mwanza, S. Iniguez, A. Gest, E. O. Smith, M. Oden, R. R. RichardsKortum, and E. Molyneux. Efficacy of a low-cost bubble CPAP system in treatment of respiratory distress in a neonatal ward in Malawi. Malawi Med J 28:131-137, 2016.

${ }^{20} \mathrm{McC}$ Cormick School of Engineering: Center for Innovation in Global Health Technologies. Northwestern University: http://www.mccormick.northwestern.edu/research/innovationglobal-health-technologies-center/, 2017.

${ }^{21}$ Mohedas, I., S. R. Daly, and K. H. Sienko. Design ethnography in capstone design: investigating student use and perceptions. Int. J. Eng. Educ. 30:888-900, 2014.

${ }^{22}$ Rice University 360 Institute for Global Health. http://www.rice360.rice.edu.

${ }^{23}$ Richards-Kortum, R., L. V. Gray, and M. Oden. IBI* series winner. Engaging undergraduates in global health technology innovation. Science 336:430-431, 2012.

${ }^{24}$ Richards-Kortum, R., Oden, M. TED Talk. https://wn. com/tedxhouston_dr._rebecca_richards_kortum_dr._maria oden.

${ }^{25}$ Shah, K. G., T. L. Slough, P. T. Yeh, S. Gombwa, A. Kiromera, Z. M. Oden, and R. R. Richards-Kortum. Novel open-source electronic medical records system for palliative care in low-resource settings. BMC Palliat Care $12: 31,2013$

${ }^{26}$ Sienko, K. H. Kathleen Sienko at TEDxUofM. https://www.youtube.com/watch?v = xxB-vAVYd7o, 2011.

${ }^{27}$ Sienko, K. H., E. E. Kaufmann, M. E. Musaazi, A. S. Sarvestani, and S. Obed. Obstetrics-based clinical immersion of a multinational team of biomedical engineering students in Ghana. Int J Gynaecol Obstet 127:218-220, 2014. 


\footnotetext{
${ }^{28}$ University of Michigan Global Health Design Initiative. http://globalhealthdesign.engin.umich.edu, 2017.

${ }^{29}$ Wilcox, L., C. Ramprasad, A. Gutierrez, M. Oden, R. Richards-Kortum, H. Sangi-Haghpeykar, and M. Gandhi. Diagnosing postpartum hemorrhage: a new way to assess blood loss in a low-resource setting. Matern Child Health $J$ 21:516-523, 2017.
}

\section{Brittany Ploss \\ Department of Biomedical Engineering \\ Duke University, Durham, NC, USA}

Tania S. Douglas

Division of Biomedical Engineering,

University of Cape Town,

Cape Town, Republic of South Africa

\section{Matthew Glucksberg}

Department of Biomedical Engineering,

Northwestern University,

Evanston, IL, USA

\section{Elsie Eah Kaufmann}

Department of Biomedical Engineering, University of Ghana,

Legon, Accra, Ghana

RoBert A. MALKin

Department of Biomedical Engineering

Duke University,

Durham, NC, USA

\section{JANET MCGRATH}

Department of Anthropology,

Case Western Reserve University,

Cleveland, OH, USA
Theresa MkandawiRe

Department of Civil Engineering,

The Polytechnic, University of Malawi,

Blantyre, Malawi

Maria Oden

Department of Biomedical Engineering,

Rice University,

Houston, TX, USA

\section{Akinniyi Osuntoki}

Department of Biochemistry,

University of Lagos,

Lagos, Nigeria

\section{ANDREw Rollins}

Department of Biomedical Engineering,

Case Western Reserve University,

Cleveland, $\mathrm{OH}$, USA

KATHLEEN Sienko

Departments of Mechanical Engineering and Biomedical Engineering,

University of Michigan,

Ann Arbor, MI, USA

ROBERT T. SSEKitoleko

Program in Biomedical Engineering,

Makerere University, Kampala, Uganda

William ReICHERT

Department of Biomedical Engineering

Duke University, Durham, NC, USA

Electronic mail: reichert@duke.edu 